\title{
Intensive care medicine in 2050: perioperative critical care
}

\author{
Zsolt Molnár ${ }^{1 *}$, Jan Benes ${ }^{2,3}$ and Daniel A. Reuter ${ }^{4}$
}

๑ 2017 Springer-Verlag Berlin Heidelberg and ESICM

When we received this invitation from Intensive Care Medicine, it initially filled us with enthusiasm but soon turned out to be a very difficult task. It is not just that we are neither fortune-tellers nor health economists, but we are also biased as we have a major interest in perioperative hemodynamic optimization [1-3], which is just one piece of the whole puzzle. On top of this we will be well within retirement age in 2050, if alive at all. But it is beyond doubt that the current generation has a huge responsibility in how medicine will look in the future. Therefore, the purpose of this paper is to highlight some aspects and trends from the present which may determine changes in the upcoming decades.

Within the next 30 years, changes in perioperative critical care will be driven by several factors, such as epidemiologic, socioeconomic, and scientific ones. However, the arising challenges will be different in various parts of the world. In developing countries-and thus affecting the majority of the world's population-many more patients than today will demand complex surgical treatments. Although the number of people who can have access to high-end medical services, considered as the gold standard in Europe and the USA, will increase, this will remain out of reach for the majority. Perhaps, an "economy of scale" also in perioperative medicine will be, at least in part, a solution in these countries: complex surgery with the need for perioperative intensive care will not be provided everywhere, but in large centers-and in dimensions much larger than those known today. And this is, from the authors' point of view, more desirable than refusing care at all to those people. The tremendous

\footnotetext{
*Correspondence: zsoltmolna@gmail.com

${ }^{1}$ Department of Anesthesiology and Intensive Care, University of Szeged,

6. Semmelweis St, Szeged 6725, Hungary
}

Full author information is available at the end of the article increase in cardiac surgery services in India serves as an example of this [4].

Developed countries will also face special challenges. Recent results of the ISOS trial demonstrated that in regards to surgical outcome-with the emphasis on quality (i.e., morbidity) - there is still room for improvement [5]. Hopefully, by 2050 medical service providers will acknowledge that reimbursement should not only depend on quantity but also on certain measures of quality, which will put (hopefully positive) pressure on perioperative intensive care [6].

The ageing patient population may also have a significant effect on intensive care in 2050. In 2005, $16.6 \%$ of Europeans were older than 65; in 2015, this proportion had already increased to $18 \%$; and in 2050 , it is estimated to reach more than 28\% [7]. Changes in America, Asia, and Africa will be similar or even more profound, and although (and also because) the overall health status of the elderly will further improve, specific perioperative treatment concepts for this particular patient group will reach critical importance.

These changes in circumstances render the need for a paradigm shift in perioperative intensive care, which has already resulted in the development of a relatively new medical field called "perioperative medicine". This should provide a more efficient and focused approach as compared to the contemporary structure in which patients are managed more or less independently by physicians, surgeons, anesthesiologists, and intensivists $[5,8]$. This concept is still developing but its potential importance will generate more reviews and opinions dedicated solely to this subject in the future. For elective high-risk cases, the use of "enhanced recovery programs" [9], homeostasis-optimizing treatments (a future step in contemporary goal-directed treatments) [10] with a combination of minimally or even non-invasive treatment modalities replacing in part contemporary extensive surgical

\section{Springer}


approaches and efforts to attenuate the undesired effects of major surgery such as postoperative hypoxemia [11] and renal dysfunction [12, 13], will aim to shorten or even omit postoperative hospitalization. Smart wearables will help in managing remote vigilance over the patient's vital function [14] and nanotechnology may improve the use of prolonged loco-regional analgesic techniques significantly, leaving patients pain-free and able to rehabilitate swiftly both enabling "at-home" postoperative course (Fig. 1) [15]. Whether robotic surgery or even machine-controlled autonomic patient care in the operating room and on the ICU will push surgeons, anesthesiologists, intensivists, and nursing staff for the most part aside, sounds scary and (hopefully) unrealistic-practicing medicine is intrinsically tied to altruism and empathy. However, in 1967 nobody thought about autonomic traffic on our roads, and a similar automatization and robotic care in perioperative medicine may actually lead to minimization of human errors in some instances. But will this make perioperative intensive care in its current form more or less unnecessary in the future? We do not believe so.

In line with this, one can hardly predict the speed at which technological developments will take place and affect our practice. But it may not be science fiction that the majority of preoperative assessments could be done via online communications or smart wearables. Highly sophisticated apps could check and analyze physiological, even laboratory parameters and perform a swift risk assessment within minutes, avoiding the need for attendance for anesthetic pre-assessment in hospitals. Genetic pretesting could play a role in predicting patients' response to major surgery, hence identifying those at high risk for developing postoperative complications. This will further open the door for even more individualized perioperative treatment strategies, bringing us away from "one size fits all" approaches. New-generation anesthetics, analgesics, and muscle relaxants with fast onset/ short half-life will be administered using automatic and partially autonomous closed loop systems applying individualized pharmacokinetic models and intelligent neural networks to tailor drug delivery to the patients' need. Contemporary methods of intraoperative monitoring will be replaced by devices enabling the analysis of multiple domains and their interactions, including complex evaluation of heart and lung function, which will be put it in the context of depth of anesthesia and degree of analgesia. Most of such monitors will be wireless, gaining information from non-invasive or semi-invasive modules and also linked to each other. Such systems will allow skilled anesthesiologists to keep more anesthetic stations under control, hence facilitate training and improve patient safety.

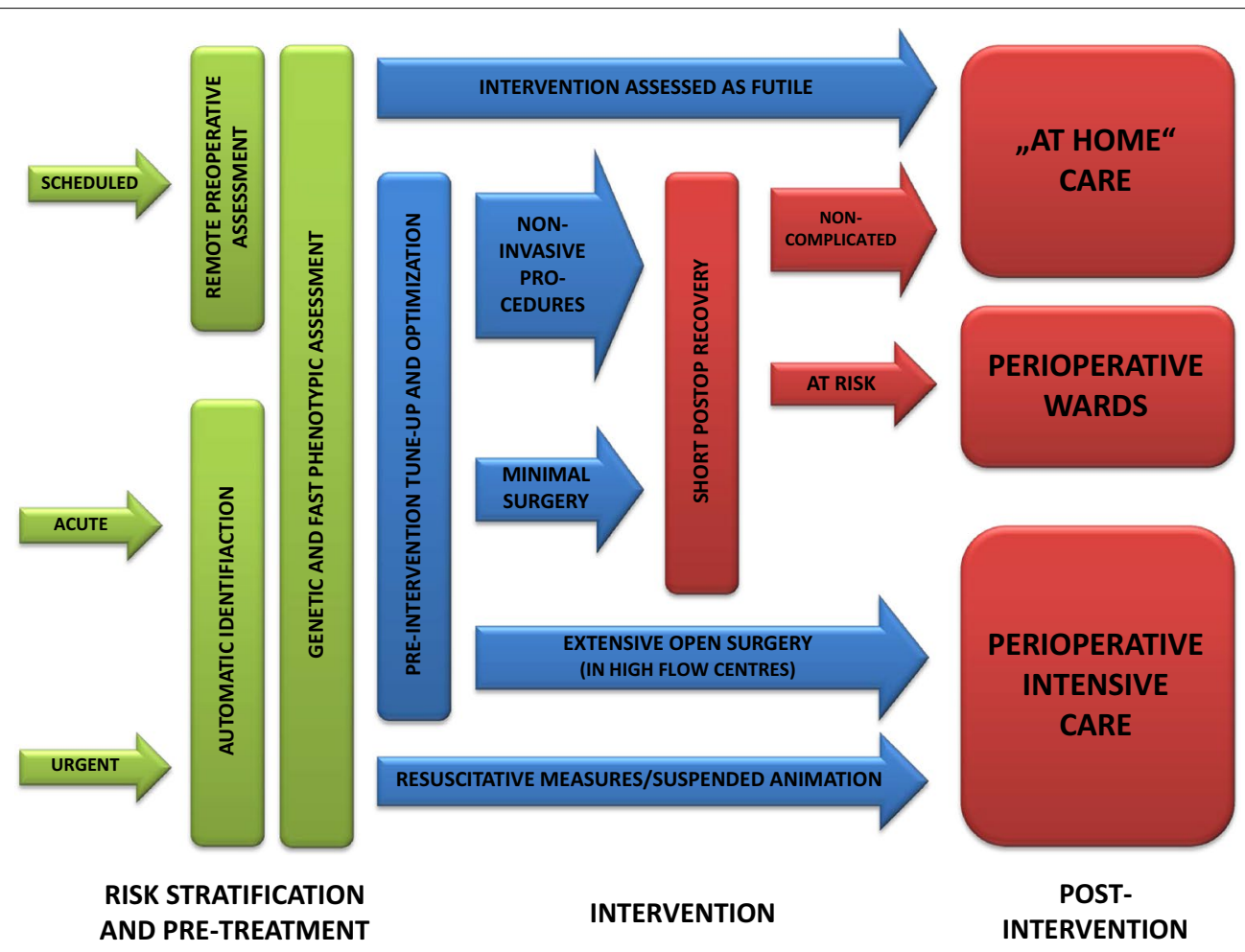

Fig. 1 Perioperative treatment pathways. Colors correspond to pre- (green), intra- (b/ue), and postoperative (red) care. See text for explanation 
Regarding major disasters, the basics of care, such as immediate resuscitation, pain relief, and damage control surgery, will most likely remain as today, but revolutionary changes may also take place, such as introducing suspended animation [16], artificial blood and tissues [17], modulating systemic inflammation, and treating molecular effects of injury on cellular or even subcellular levels [18]. But of course, every solution for a certain problem will inevitably bring up new ones. Therefore, every new development will have its own side effects and risks, which will have to be solved by the generations coming after 2050.

Finally, in addition to trying to evaluate current trends and predict how perioperative critical care will look (Supplementary Table 1), we also asked ourselves how we would like it to be in 2050 ? It may be completely different compared to what we have predicted, but one thing should not change and it is of utmost importance: the quality of caring staff with the patient in focus. Nothing, not even hi-tech autonomous robotic systems, should ever replace the devoted, well-trained, thinking, and caring physicians and nurses either today or in 2050. Hence the motto should remain the same: "And as you wish that others would do to you, do so to them". (Luke 6,31).

\section{Electronic supplementary material}

The online version of this article (doi:10.1007/s00134-017-4703-6) contains supplementary material, which is available to authorized users.

\section{Author details}

1 Department of Anesthesiology and Intensive Care, University of Szeged, 6. Semmelweis St, Szeged 6725, Hungary. ${ }^{2}$ Department of Anesthesiology and Intensive Care, Faculty of Medicine in PIzen, Charles University, alej Svobody 80, 30640 Plzen, Czech Republic. ${ }^{3}$ Biomedical Centre, Faculty of Medicine in Plzen, Charles University, alej Svobody 76, 32300 Plzen, Czech Republic. ${ }^{4}$ Center of Anesthesiology and Intensive Care Medicine, Hamburg Eppendorf University Medical Center, Martinistr. 52, 20246 Hamburg, Germany.

Received: 4 January 2017 Accepted: 27 January 2017 Published online: 8 February 2017

\section{References}

1. Salzwedel C, Puig J, Carstens A et al (2013) Perioperative goal-directed hemodynamic therapy based on radial arterial pulse pressure variation and continuous cardiac index trending reduces postoperative complications after major abdominal surgery: a multi-center, prospective, randomized study. Crit Care 17:R191
2. Benes J, Chytra I, Altmann P et al (2010) Intraoperative fluid optimization using stroke volume variation in high risk surgical patients: results of prospective randomized study. Crit Care 14:R118

3. Mikor A, Trasy D, Nemeth MF et al (2015) Continuous central venous oxygen saturation assisted intraoperative hemodynamic management during major abdominal surgery: a randomized, controlled trial. BMC Anesthesiol 15:82. doi:10.1186/s12871-015-0064-2

4. Govindarajan V, Ramamurti R (2013) India's secret to low cost health care. Harvard Business Review. https://hbr.org/2013/10/indias-secret-to-lowcost-health-care. Accessed 19 Dec 2016

5. The International Surgical Outcomes Study group (2016) Global patient outcomes after elective surgery: prospective cohort study in 27 low-, middle- and high-income countries. Br J Anaesth 117:601-609

6. Porter ME, Lee TH (2013) The strategy that will fix healthcare. Harvard Business Review. https://hbr.org/2013/10/the-strategy-that-will-fixhealth-care. Accessed 19 Dec 2016

7. European Commission. Eurostat: http://ec.europa.eu/eurostat/statisticsexplained. Accessed 19 Dec 2016

8. Grocott MP, Pearse RM (2012) Perioperative medicine: the future of anaesthesia? Br J Anaesth 108:723-726

9. Thiele RH, Raghunathan K, Brudney CS et al (2016) American Society for Enhanced Recovery (ASER) and Perioperative Quality Initiative (POQI) joint consensus statement on perioperative fluid management within an enhanced recovery pathway for colorectal surgery. Perioper Med 5:24. doi:10.1186/s13741-016-0049-9

10. Futier E, Paugam-Burtz C, Godet T, Khoy-Ear L, Rozencwajg S, Delay JM, Verzilli D, Dupuis J, Chanques G, Bazin JE, Constantin JM, Pereira B, Jaber S, OPERA study investigators (2016) Effect of early postextubation highflow nasal cannula vs conventional oxygen therapy on hypoxaemia in patients after major abdominal surgery: a French multicentre randomised controlled trial (OPERA). Intensive Care Med 42:1888-1898

11. Tanczos K, Nemeth M, Molnar Z (2015) What's new in hemorrhagic shock? Intensive Care Med 41:712-714

12. O'Connor ME, Kirwan CJ, Pearse RM, Prowle JR (2016) Incidence and associations of acute kidney injury after major abdominal surgery. Intensive Care Med 42:521-530

13. Mårtensson J, Bellomo R (2015) What's new in perioperative renal dysfunction? Intensive Care Med 41:514-516

14. Michard F (2016) Hemodynamic monitoring in the era of digital health. Ann Intensive Care 6:15. doi:10.1186/s13613-016-0119-7

15. Mantha VRR, Nair HK, Venkataramanan R et al (2014) Nanoanesthesia: a novel, intravenous approach to ankle block in the rat by magnet-directed concentration of ropivacaine-associated nanoparticles. Aesth Analg 118:1355-1362

16. Asfar P, Radermacher P (2015) Drug-induced "suspended animation": can a dream come true? Crit Care Med 43:1528-1530

17. Chang TMS (2012) From artificial blood cells, oxygen carriers, and oxygen therapeutics to artificial cells, nanomedicine and beyond. Artif Cells Blood Substit Immobil Biotechnol 40:197-199

18. Dumont CM, Park J, Shea LD (2015) Controlled release strategies for modulating immune responses to promote tissue regeneration. J Control Release 219:155-166 